\title{
Optimization Decision Method for Procuring Divisible Goods
}

\author{
Congjun Rao* \\ College of Mathematics and computer Science, Huanggang Normal University \\ Huanggang 438000, P. R. China \\ Institute of Systems Engineering, Huazhong University of Science and Technology \\ Wuhan 430074, P. R. China \\ Xinlin Wu \\ Institute of Systems Engineering, Huazhong University of Science and Technology \\ Wuhan 430074, P. R. China
}

Received 5 April 2010

Accepted 16 August 2012

\begin{abstract}
The procurement decision problem of divisible goods is investigated, and an optimization decision method for procuring a kind of divisible goods (such as coal, oil, electricity, gas, etc.) is presented based on multi-attribute auction. Firstly, the procurement decision problem of divisible goods is converted into a multi-attribute auction of divisible goods. Correspondingly, several important assumptions of multi-attribute procurement auction are given, and the buyer's utility function and the supplier's utility function are defined. Secondly, the scoring rules and bidding rules are given. And aiming at maximizing the buyer's expected utility, an optimization decision model of selecting the suppliers in multi-attribute auction is established. By solving this model, the optimal suppliers and the optimal allocation strategies are obtained. Thirdly, the suppliers' optimal bidding strategies are discussed, and the feasibility of multi-attribute auction mechanism is proved. Lastly, a multi-attribute auction example about the electric coal procurement is given to demonstrate the availability and rationality of our procurement decision method.
\end{abstract}

Keywords: Divisible goods procurement, Optimization decision model, Multi-attribute auction, Electric coal procurement

\section{Introduction}

As a price discovery and resource allocation mechanism, Auction has been widely used in many areas of the electronic commerce. Nowadays, more and more enterprises use reverse auction mechanism for electronic procurement. However, most procurement mechanisms are proposed only on the base of the traditional single price auction without considering other non-price attributes (such as development cycle, delivery time, different quality parameters, service quality, and suppliers' reputation). It has seriously restricted the further applications for auctions in electronic commerce[1]. In practice electronic commerce, the procurement actives usually involve many other dimensions in addition to the price[2-6]. For example, in the electric coal procurement, the buyer will consider the contract which is composed of multiple attributes, such as quality (Tvdaf, calorific value, moisture, ash value, ash melting point and coal sulfur value), quantity, transport capacity, suppliers' credibility, price, and so on. We call this kind of procurement under the multiattribute information as a multi-attribute procurement. Multi-attribute auctions provide us with a valid and feasible way to solve the problem of multi-attribute procurement. Practice has proved that multi-attribute auction is a short-term and efficient procurement mechanism[5-9]. Bichler[10] defined multi-attribute auctions as a class of market mechanisms, which

*Corresponding Author: cjrao@163.com 
enables automated negotiation on multiple attributes of a deal. This feature is especially useful in procurement auctions where buyers negotiate with multiple suppliers over heterogeneous or homogeneous goods. Multiattribute auctions are also called multidimensional auctions or multi-criteria auctions[11-12].

On the study of multi-attribute auctions, Thiel[11] first gave a partial analysis on multi-attribute auctions. He showed that the problem of designing optimal multidimensional auctions will be equivalent to the design of unidimensional auctions. Based on Thiel's work, Che[2] gave a thorough analysis of the design of multi-attribute auctions. He derived a two-dimensional version of the revenue equivalence theorem, and also designed an optimal scoring rule based on the assumption that the buyer knows the probability distribution of the supplier's cost parameter. Thereafter, Branco[12] extended Che's work. His analysis was based on Che's independent cost model and derived an optimal auction mechanism for the case when the bidding firms' costs are correlated. The works of Che and Branco were among the first considering multidimensional auctions. But both were studying auction design only in a two issues context, i.e., price and quality.

Moreover, interest and need from e-procurement companies in designing novel multi-attribute auction mechanisms is growing. For example, Mishra and Veeraman[13] first brought multi-attribute auctions into the area of procurement economy. He proposed a reverse auction algorithm for the outsourcing economy and proved that this reverse auction algorithm achieves nearly efficient allocation and the final prices are also nearly competitive. Later, Beil and Wein[14] focused on buyer utility-maximization in multi-attribute auctions, i.e., optimal auction design based on the Chief Technology Officer of Frictionless Commerce, who was seeking help with designing a new automated eRFQ (Request for Quotation) mechanism. They suggested an inverse-optimization based approach that allows the buyer via several changes in the announced scoring rule, to learn the suppliers' cost functions and then determine a scoring rule that maximizes the buyer's utility within an open ascending auction format. Parallel to these works, David et al.[15] extended Che's model for more realistic electronic commerce applications, where the reverse auction considers multiple non-price attributes. For this case they analyzed the first-score, the secondscore and the English auctions.

Multi-attribute auction mechanisms are more suitable than classical price-based mechanisms since the underlying value of the transaction does not depend just on price. From this perspective, Chen-Ritzo et al.[16] presented an ascending auction mechanism for a buyer whose utility function is known and dependent on three attributes(quality, lead time and price) motivated by a supply chain management problem setting. Perrone et al.[17] provided a project management approach for multi-attribute auction design for standardized engineering services procurement in the context of new product development in automotive industry. They argued that price and time based auctions can also work as a mechanism to increase the reliability of engineering planning in that they allow involvement of suppliers in determining variables such as duration and cost of a given activity which are to be used to construct and manage the whole project plan. In addition, Ray et al. [18] proposed a novel multi-attribute relationshippreserving reverse auction mechanism for a limited supplier base. The mechanism enables healthy competition among the suppliers by retaining them in the supplier base. The simulation study interestingly shows that a buyer derives higher utility by using the proposed mechanism as compared to the existing mechanism where no incentive is provided to the suppliers. Karakaya and Köksalan[19] introduced an interactive approach to provide aid both to the buyer and the sellers for a multi-attribute, single-item, multiround, reverse auction. The experiments show that the mechanism supports the sellers well and the winning bids are very close to the bids that would have been obtained under full information. Liu et al.[20] analyzed multi-attribute procurement auctions with risk-averse suppliers. They showed that as the number of suppliers increases or the suppliers become more risk-averse, the equilibrium bidding price decreases under the first-score auction but remains the same under the second-score auction.

From the existing important research of multiattribute auctions we can conclude that most results are obtained by considering the multi-attribute auctions for indivisible goods, and the bid winner is only one. The research on the multi-attribute auctions for divisible goods which aims at the characteristic of homogeneousness and divisibility is very few. In this paper, the procurement decision problem of divisible goods (such as coal, oil, electricity, gas, etc.) is studied, and a procurement decision model is presented based on multi-attribute auction. In this optimization decision 
model, other attributes in addition to the price and quantity are considered further, such as the development cycle, delivery time, different quality parameters, service quality, and suppliers' reputation, and so on. Specially, in the traditional models, it is supposed all bidders are symmetrical, and the buyers' utility functions in are too special to meet the generality features, and the fact that some attributes' marginal production costs of the seller are increasing is not satisfied. Therefore, in this paper, we suppose all bidders are asymmetrical, and improve the utility functions of buyers and sellers. Compare with traditional models (e.g. David[15]), our model is more realistic, and has a broader applicability.

The rest of the paper is organized as follows. In section 2, we describe the problem of multi-attribute procurement for divisible goods, and then convert it to a multi-attribute auction. Section 3 presents an optimization decision method for procuring divisible goods based on multi-attribute auction. Section 4 gives an application example about the electric coal procurement to show the availability and rationality of our procurement decision method. In Section 5, a conclusion summarizes our work and gives directions for future researches.

\section{Problem Description and Transformation}

First of all, we describe the problem of multi-attribute procurement.

Suppose that one buyer will procure $Q_{0}$ units of a divisible good (e.g. coal, oil, electricity, gas, etc.) from $n \quad(n \geq 2)$ suppliers. All suppliers will submit the trading information including price, quantity, quality, delivery time and so on to the buyer after the buyer announcing the purchasing information. Then the buyer will select the optimal suppliers and allocate the allowable supply quantities of the procurement goods to them according to the submitted information.

In a multi-attribute procurement, the buyer's objective is to maximize his utility and to maximize the allocation efficiency. In fact, each supplier's submitted information is private information, and is complicated and asymmetric. So the facticity of this information can not be guaranteed, and the goals of utility maximization and allocation efficiency maximization are difficult to achieve. Thus, the buyer must create an incentive and competitive trading environment to induce suppliers to announce their actual information truthfully. However, the traditional procurement methods can not achieve this result. To present an incentive and competitive method to procuring a kind of divisible goods, we propose an optimization decision method based on multi-attribute auction theory in this paper. Next we give the process of converting a multi-attribute procurement to a multi-attribute auction.

Suppose that the buyer is an auctioneer, he wants to buy $Q_{0}$ units of a divisible good. The $n(n \geq 2)$ suppliers are regarded as $n$ potential bidders, the set of bidders is denoted as $N=\{1,2, \ldots, n\}$. We suppose that all bidders are risk neutral, and all want to maximize their expected utilities. In the auction, the auctioned goods are described by multiple attributes, i.e., price, quantity, quality, and so on. Let the attribute set be $A=\left\{p, q, A_{1}, A_{2}, \mathrm{~L}, A_{m}\right\}$, where $p$ is the price attribute, $q$ is the quantity attribute, and $A_{1}, A_{2}, \mathrm{~L}, A_{m}$ are $m$ attributes in addition to the price and quantity. These $m$ attributes are called quality attributes, such as the development cycle, delivery time, different quality parameters, service quality, and suppliers' reputation, and so on. To simplify the analysis, we suppose that the $m$ quality attributes are continuous, nonnegative variables (thus the domains of the cost and utility functions are the nonnegative real numbers) and that larger values of $a_{i 1}, a_{i 2}, \mathrm{~L}, a_{i m}$ are more desirable from the auctioneer's point of view and more costly from the bidders' point of view [15]. In the bidder $i$ 's bidding, the values of attribute $p, q, A_{1}, A_{2}, \mathrm{~L}, A_{m}$ are denoted as $p_{i}, q_{i}, a_{i 1}, a_{i 2}, \mathrm{~L}, a_{i m}$ respectively.

The main idea of multi-attribute auction of divisible goods is represented as follows. At the beginning of the auction, the auctioneer announces the basic requirement and the scoring rules for the procurement good to all bidders. Then $n$ bidders submit biddings in the form of $\left(p_{i}, q_{i}, a_{i 1}, a_{i 2}, \mathrm{~L}, a_{i m}\right)$ as a sealed bidding. Within the auction, every bidder has opportunity to submit multiple rounds biddings. According to the bidders' biddings, the auctioneer publishes the scores and rank order in time, but does not reveal the bidders' identities or detailed biddings. Through multiple rounds of bidding, the bidding process is over. Then the auctioneer will select the optimal bidders by aiming at maximizing his 
expected utility, and allocate the allowable supply quantities to the winners. The allowable supply quantity to bidder $i$ is denoted as $q_{i}^{*}$, which satisfy $\sum_{i=1}^{n} q_{i}^{*}=Q_{0}$, where $q_{i}^{*} \geq 0, i=1,2, \mathrm{~L}, n$.

\section{Optimization Decision Method Based on Multi- attribute Auction}

\subsection{The utility functions of bidders and auctioneer}

Suppose that the bidder $i$ 's cost function is additive across attributes[15], and the cost function on the one unit of a divisible good with the quality $a_{i}=\left(a_{i 1}, a_{i 2}\right.$, $\left.\mathrm{L}, a_{i m}\right)$ is denoted as

$$
c_{i}^{1}=\sum_{j=1}^{m} c_{i j}\left(s_{i}, a_{i j}\right),
$$

where $s_{i}$ is the bidder $i$ 's cost efficiency parameter (hereinafter referred to as the bidder $i$ 's type). The set of the bidder s' types is denoted as $s=\left(s_{1}, s_{2}, \mathrm{~L}, s_{n}\right) . s_{i}$ is the bidder $i$ 's private information about the costs of improving the quality of the product it sells, and is only known by bidder $i$, other bidders do not know the real value of $s_{i}$ and treat it as a draw from a cumulative distribution $F_{i}\left(s_{i}\right) . F_{i}\left(s_{i}\right)$ is defined on a support $S=[\underline{s}, \bar{s}]$ and with a density function $f_{i}\left(s_{i}\right)$. Any two variables $s_{i}$ and $s_{j}$ with $i \neq j$ are independent. $c_{i j}\left(s_{i}, \cdot\right)$ is the bidder $i$ 's cost function on the quality attribute $A_{j}(j=1,2, \mathrm{~L}, m)$. Function $c_{i j}\left(s_{i},{ }^{\circ}\right)$ is increasing, convex and twice continuously differentiable in $a_{i j}$, and $c_{i j}\left(s_{i}, 0\right)=0$, and increasing in type $s_{i} \cdot c_{i j}\left(s_{i}, \cdot\right)$ is the bidder $i$ 's private information, and is only known by bidder $i$ himself.

Based on the cost function $c_{i}^{1}=\sum_{j=1}^{m} c_{i j}\left(s_{i}, a_{i j}\right)$, the utility of bidder $i$ who submit the unit price $p_{i}$ and gets the one unit of a divisible good can be expressed as

$$
\begin{aligned}
& u_{s i}^{1}\left(s_{i}, p_{i}, a_{i 1}, a_{i 2}, \mathrm{~L}, a_{i m}\right) \\
& =p_{i}-c_{i}^{1}=p_{i}-\sum_{j=1}^{m} c_{i j}\left(s_{i}, a_{i j}\right)
\end{aligned}
$$

In addition, the auctioneer's utility function is assumed to be additive across quality attributes $A_{1}, A_{2}, \mathrm{~K}, A_{m}$. When the bidder $i$ with the bid $\left(p_{i}, q_{i}, a_{i 1}, a_{i 2}, \mathrm{~L}, a_{i m}\right)$ wins the bid, the revenue of one unit good to auctioneer is defined as

$$
V_{i}^{1}=\sum_{j=1}^{m} v_{j}\left(a_{i j}\right)
$$

where $v_{j}(\cdot)$ is the revenue function on attribute $A_{j}(j=1,2, \mathrm{~L}, m) . v_{j}(\cdot)$ is increasing, concave and twice continuously differentiable in $a_{i j} . v_{j}(\cdot)$ is the same to all bidders, and is public information which will be announced at the beginning of the auction. Based on the revenue function $V_{i}^{1}$, the auctioneer's utility of one unit good from the bidder $i$ can be expressed as

$$
\begin{aligned}
& u_{b_{i}}^{1}\left(p_{i}, q_{i}, a_{i 1}, a_{i 2}, \mathrm{~L}, a_{i m}\right) \\
& =V_{i}^{1}-p_{i}=\sum_{j=1}^{m} v_{j}\left(a_{i j}\right)-p_{i}
\end{aligned}
$$

For above utility functions of bidders and auctioneer, the traditional model (e.g. David[15]) supposed all bidders are symmetrical, and defined the auctioneer's utility function as

$$
u_{b}^{1}=\sum_{j=1}^{m} v_{j}\left(a_{i j}\right)-p_{i}=\sum_{j=1}^{m} w_{j} \sqrt{a_{i j}}-p_{i}
$$

where $w_{j}$ is the weight of attribute $A_{j}$ given by the auctioneer. In this utility function, $v_{j}\left(a_{i j}\right)=w_{j} \sqrt{a_{i j}}$, this assumption is very special, and lacks generality. Moreover, David defined each bidder's utility function as

$$
\begin{aligned}
& u_{s i}^{1}\left(s_{i}, p_{i}, a_{i 1}, a_{i 2}, \mathrm{~L}, a_{i m}\right) \\
& =p_{i}-\sum_{j=1}^{m} c_{i j}\left(s_{i}, a_{i j}\right) \\
& =p_{i}-s \sum_{j=1}^{m} k_{j} a_{i j}
\end{aligned}
$$

where $k_{j}$ is the quality attribute coefficient of the $j$ th attribute, and $s=s_{1}=s_{2}=\mathrm{L}=s_{n}$ is each bidder's cost efficiency parameter. In fact, this assumption $c_{i}^{1}=\sum_{j=1}^{m} c_{i j}\left(s_{i}, a_{i j}\right)=s \sum_{j=1}^{m} k_{j} a_{i j}$ does not satisfy the fact that some attributes' marginal production costs of the bidder (supplier) are increasing.

In addition, in practice of auction, bidders are asymmetrical, so $s=s_{1}=s_{2}=\mathrm{L}=s_{n}$ is usually not satisfied. In order to overcome the deficiency of the traditional models, we propose improved utility functions of bidders and auctioneer as follows. 
1) The auctioneer's utility of one unit good from the bidder $i$ is rewritten as

$$
\begin{aligned}
& u_{b i}^{1}\left(p_{i}, q_{i}, a_{i 1}, a_{i 2}, \mathrm{~L}, a_{i m}\right) \\
& =V_{i}^{1}-p_{i}=\sum_{j=1}^{m} w_{j} a_{i j}^{l_{j}}-p_{i}
\end{aligned}
$$

where $0<l_{j} \leq 1$, to ensure the marginal utility of attribute $A_{j}$ to auctioneer is nonincreasing.

2) The utility of bidder $i$ who submit the unit price $p_{i}$ and gets the one unit of a divisible good can be rewritten as

$$
\begin{aligned}
& u_{s i}^{1}\left(s_{i}, p_{i}, a_{i 1}, a_{i 2}, \mathrm{~L}, a_{i m}\right) \\
& =p_{i}-\sum_{j=1}^{m} c_{i j}\left(s_{i}, a_{i j}\right) \\
& =p_{i}-\sum_{j=1}^{m} s_{i} k_{j} a_{i j}^{h_{j}}
\end{aligned}
$$

where $h_{j} \geq 1$, to ensure the marginal cost of attribute $A_{j}$ to bidder is nondecreasing.

Utility functions (1) and (2) are presented based on the assumption that all bidders are asymmetrical. Compare with traditional models (e.g. David[15]), our utility functions (1) and (2) are more realistic, and has a broader applicability.

Suppose the bidder $i$ with the bid $\left(p_{i}, q_{i}, a_{i 1}, a_{i 2}\right.$, $\mathrm{L}, a_{i m}$ ) wins the bid, and he gets the allowable supply quantity $q_{i}^{*}$. Based on the utility functions (1) and (2), the total utility of bidder $i$ who submit the unit price $p_{i}$ and gets the allowable supply quantity $q_{i}^{*}$ can be expressed as

$$
\begin{aligned}
& u_{s i}\left(s_{i}, p_{i}, q_{i}, a_{i 1}, a_{i 2}, \mathrm{~L}, a_{i m}\right) \\
& =q_{i}^{*} u_{s i}^{1}\left(s_{i}, p_{i}, a_{i 1}, a_{i 2}, \mathrm{~L}, a_{i m}\right) \\
& =q_{i}^{*}\left(p_{i}-\sum_{j=1}^{m} s_{i} k_{j} a_{i j}^{h_{j}}\right)
\end{aligned}
$$

and the auctioneer's utility from bidder $i$ can be expressed as

$$
\begin{aligned}
& u_{b i}\left(p_{i}, q_{i}, a_{i 1}, a_{i 2}, \mathrm{~L}, a_{i m}\right) \\
& =q_{i}^{*} u_{b i}^{1}\left(p_{i}, q_{i}, a_{i 1}, a_{i 2}, \mathrm{~L}, a_{i m}\right) \\
& =q_{i}^{*}\left(\sum_{j=1}^{m} w_{j} a_{i j}^{l_{j}}-p_{i}\right)
\end{aligned}
$$

When the auction is over, the auctioneer's total utility is

$$
\begin{aligned}
u_{b} & =\sum_{i=1}^{n} u_{b i}\left(p_{i}, q_{i}, a_{i 1}, a_{i 2}, \mathrm{~L}, a_{i m}\right) \\
& =\sum_{i=1}^{n} q_{i}^{*}\left(\sum_{j=1}^{m} w_{j} a_{i j}^{l_{j}}-p_{i}\right)
\end{aligned}
$$

\subsection{Scoring function}

Scoring function is a function which is announced to all bidders at the beginning of the auction to select the optimal biddings. Since the scoring function is given by the auctioneer, he must achieve the goal of utility maximization, i.e., $\max u_{b}=\sum_{i=1}^{n} q_{i}^{*}\left(\sum_{j=1}^{m} w_{j} a_{i j}^{l_{j}}-p_{i}\right)$, which is obtained from (5).

In utility function $u_{b}$, we denote $\sum_{j=1}^{m} w_{j} a_{i j}^{l_{j}}-p_{i}=S_{i}$.

We can easily conclude that when $q_{i}^{*}$ is unchanged, $u_{b}$ is increasing in $S_{i}$, and when $p_{i}$ is unchanged, the value of $S_{i}$ increases with the increase of the quality level. Based on these conclusions, to achieve the goal of maximizing utility and to induce bidders to announce their actual costs truthfully, we define the scoring function which is announced to bidders as

$$
S_{i}=\sum_{j=1}^{m} w_{j} a_{i j}^{l_{j}}-p_{i}
$$

\subsection{Basic rules}

Firstly, in order to procure high-quality goods and achieve the goal of utility maximization, the auctioneer will set reserve price, reserve quantity and reserve score which are announced to all bidders at the beginning of the auction. Concretely, for one unit good, the auctioneer will set a maximum price limitation $\bar{p}$ and a minimum quality standards $\underline{a}=\left(\underline{a}_{1}, \underline{a}_{2}, \mathrm{~L}, \underline{a}_{m}\right)$, which means that the bidders' bidding prices in each round bidding must be less or equal to $\bar{p}$, and the bidders' quality level must be not inferior to $\underline{a}=\left(\underline{a}_{1}, \underline{a}_{2}, \mathrm{~L}, \underline{a}_{m}\right)$, i.e., the value $a_{j}$ of attribute $A_{1}$ must satisfy $a_{j} \geq \underline{a}_{j}$. From $\bar{p}$ and $\underline{a}$, there is a minimum score (reserve score) $\underline{S}=\sum_{j=1}^{m} w_{j}\left(\underline{a}_{j}^{l_{j}}\right)-\bar{p}$, which means all bidders' score in each round of bidding must be greater than or 
equal to $\underline{S}$. The above conditions must be satisfied, otherwise, the bidder's bidding is regarded as invalid biddings.

Secondly, in order to speed up the auction process and improve the auction efficiency, here we present a minimum bid increment method. Let bidder $i$ 's bidding in the $(t+1) t h$ round be $\left(p_{i}^{(t+1)}, q_{i}^{(t+1)}, a_{i 1}^{(t+1)} a_{i 2}^{(t+1)}\right.$, $\left.\mathrm{L}, a_{i m}^{(t+1)}\right)$, then the bidder $i$ 's score in the $(t+1) t h$ round bidding can be denoted as

$$
S_{i}^{(t+1)}=\sum_{j=1}^{m} w_{j}\left(a_{i j}^{(t+1)}\right)^{l_{j}}-p_{i}^{(t+1)} .
$$

To ensure the bid $\left(p_{i}^{(t+1)}, q_{i}^{(t+1)}, a_{i 1}^{(t+1)} a_{i 2}^{(t+1)}, \mathrm{L}, a_{i m}^{(t+1)}\right)$ of bidder $i$ with type $s_{i}$ is valid, the score $S_{i}^{(t+1)}$ must be equal to or higher than the current highest score $S_{\max }^{\text {current }}$. We suppose there is a minimum bid increment $D(D>0)$, namely, the bidder $i$ 's score of the $(t+1) t h$ round bidding is

$$
S_{i}^{(t+1)}=\sum_{j=1}^{m} w_{j}\left(a_{i j}^{(t+1)}\right)^{l_{j}}-p_{i}^{(t+1)}=S_{\max }^{\text {current }}+D .
$$

When the bidder $i$ is no longer to submit a new round of bidding, which means he finishes his bidding. When all bidders are no longer to submit new biddings, the whole bidding process is over.

Thirdly, we give the payment rules. When the auction is over, each winner gets the supply contract. In the supply contract, the transaction unit price is just the bid price in winner's final bidding, and the quality level of the supply goods is just the bidder's promised quality in his final bidding.

Finally, in order to meet the needs of the practical procurement, we can also set some special rules, for example, (i) The limitation of the bidders' maximum bid quantities i.e., $q_{i} \leq q_{\max }, i=1,2, \mathrm{~L}, n$, where $q_{\max }$ is the limitative maximum bid quantity for all bidders. The purpose of this rule is to seek more partners and build more extensive cooperative relations for buyers, and to let more bidders have the chance to win the bid. This rule will be announced to all bidders at the beginning of the auction by the auctioneer. (ii) The limitation of delivery time. The bidder's delivery time $T_{i}$ must satisfy $T_{i} \leq T$, where $T$ is the longest delivery time. The purpose of this rule is to shorten the procurement time and improve the efficiency of procurement. (iii) Each bidder's supply quantity $q_{i}$ is stipulated a fixed value $Q_{i}^{*}, i=1,2, \mathrm{~L}, n$, which means is not allowed to change in all rounds of bidding. $Q_{i}^{*}$ is determined by the bidder $i$ according to his production ability within the contractual time and maximum bid quantity $q_{\max }$ given by the auctioneer, and is bidder $i$ 's private information.

\subsection{Winner selection model}

In this paper, the procuring good which we consider is a kind of divisible goods. In practical procurement, the goods are in great demand. The quantity of the good is provided by single bidder is often limited. It is difficult to meet the buyer's needs within the stipulated time. To solve this problem, the buyer can use the multi-source procurement strategy. Under this strategy, the winner may be not unique, can be more than one.

Next we will establish the winner selection model.

From section 2, the auctioneer's goal is to maximize his utility, i.e.,

$$
\max u_{b}=\sum_{i=1}^{n} q_{i}^{*}\left(\sum_{j=1}^{m} w_{j} a_{i j}^{l_{j}}-p_{i}\right),
$$

To achieve this goal, the following conditions must be satisfied.

1) The sum of the allowable supply quantities for all winners is $Q_{0}$, i.e.,

$$
\sum_{i=1}^{n} q_{i}^{*}=Q_{0}
$$

where $q_{i}^{*}$ is less than or equal to the submitted bid quantity $q_{i}$ and is nonnegative, together with the limitation given by section 3.3 , we have $0 \leq q_{i}^{*} \leq q_{i} \leq q_{\max }$.

2) For the bid $\left(p_{i}, q_{i}, a_{i 1}, a_{i 2}, \mathrm{~L}, a_{i m}\right), i=1,2, \mathrm{~L}, n$, the auctioneer's utility and the bidders' utilities must be nonnegative, i.e.,

$$
\begin{gathered}
u_{b i}=q_{i}^{*}\left(\sum_{j=1}^{m} w_{j} a_{i j}^{l_{j}}-p_{i}\right) \geq 0, \\
u_{s i}=q_{i}^{*}\left(p_{i}-\sum_{j=1}^{m} s_{i} k_{j} a_{i j}^{h_{j}}\right) \geq 0, \\
i=1,2, \mathrm{~L}, n
\end{gathered}
$$

Based on above discussion, the winner selection model in multi-attribute procurement can be expressed as 
$\max u_{b}=\sum_{i=1}^{n} q_{i}^{*}\left(\sum_{j=1}^{m} w_{j} a_{i j}^{l_{j}}-p_{i}\right)$,

$$
\begin{cases}\text { s.t. } & \\ \sum_{i=1}^{n} q_{i}^{*}=Q_{0} & \\ 0 \leq q_{i}^{*} \leq q_{i} \leq q_{\max }, & i=1,2, \mathrm{~L}, n \\ u_{s i} \geq 0, & i=1,2, \mathrm{~L}, n \\ u_{b i} \geq 0, & i=1,2, \mathrm{~L}, n\end{cases}
$$

When all bidders finish their biddings through multiple rounds bidding, $p_{i}, q_{i}, a_{i j}$ are all known numbers, and $Q_{0}, w_{j}, l_{j}$ are all public information which is announced to all bidders at the beginning of the auction by the auctioneer. By substituting these known values into $M_{1}$, and solving $M_{1}$, the allowable supply quantity $q_{i}^{*}(i=1,2, \mathrm{~L}, n)$ can be obtained.

At the beginning of the auction, the auctioneer may announce the method of selecting winners described by $M_{1}$ to all bidders. From $M_{1}$, the bidder $i$ 's allowable supply quantity $q_{i}^{*}$ increases with the increase of the bidder $i$ 's score $S_{i}=\sum_{j=1}^{m} w_{j} a_{i j}^{l_{j}}-p_{i}$, so $M_{1}$ can induce bidders to announce their actual costs truthfully to raise their scores.

\section{Optimal Strategy Analysis}

After giving the multi-attribute auction mechanism, we analyze the bidders' optimal bidding strategy in this section. Suppose that all bidders use the best-response strategy (BRS) to bid. The main idea of BRS is that a bidder chooses his next bidding to maximize his current utility, assuming no other bidders change their biddings. Let the minimum bid increment be $D$, and the current highest score be $S_{\max }^{\text {current }}$. If the bidder $i$ will bid in the $(t+1) t h$ round, then his optimal bidding strategy $\left(p_{i}^{(t+1)^{*}}, q_{i}^{(t+1)^{*}}, a_{i 1}^{(t+1)^{*}} a_{i 2}^{(t+1)^{*}}, \mathrm{~L}, a_{i m}^{(t+1)^{*}}\right)$ is the solution of the optimization problem $M_{2}$ as follows.

$$
\max u_{s i}^{1}=p_{i}^{(t+1)}-\sum_{j=1}^{m} s_{i} k_{j}\left(a_{i j}^{(t+1)}\right)^{h_{j}}
$$

$\left(M_{2}\right) \quad$ s.t.

$$
S_{i}^{(t+1)}=\sum_{j=1}^{m} w_{j}\left(a_{i j}^{(t+1)}\right)^{l_{j}}-p_{i}^{(t+1)}=S_{\max }^{\text {current }}+D
$$

Note that the bidder $i$ 's supply quantity $q_{i}^{(t+1)^{*}}$ is a fixed value by the rules in section 3.3. That is, it is the same in all rounds of bidding, i.e., $q_{i}^{(t+1)^{*}}=q_{i}^{(t)^{*}}=\mathrm{L}=q_{i}^{(1)^{*}}=Q_{i}^{*}$. Thus, when considering the optimal bidding strategies of bidders, we just only consider the optimal value $p_{i}^{(t+1)^{*}}$ of the price attribute and the optimal value $a_{i j}^{(t+1)^{*}}$ of the quality attribute.

By analyzing $M_{2}$, the optimal values $p_{i}^{(t+1)^{*}}$ and $a_{i j}^{(t+1)^{*}}$ can be deduced. They are given in the following Proposition 1.

Proposition 1. Suppose that the current highest score is $S_{\max }^{\text {current }}$, the minimum bid increment is $D$, the auctioneer's utility function and bidder $i$ 's utility function given by (1) and (2), and the scoring function $S_{i}$ given (5). Then the optimal bidding strategy of bidder $i$ is as follows.

(i) If the following condition is satisfied

$$
\begin{gathered}
\sum_{j=1}^{m} w_{j}\left(\frac{s_{i} k_{j} h_{j}}{l_{j} w_{j}}\right)^{\frac{l_{j}}{l_{j}-h_{j}}}-S_{\max }^{\text {current }}-D-\sum_{j=1}^{m} s_{i} k_{j}\left(\frac{s_{i} k_{j} h_{j}}{l_{j} w_{j}}\right)^{\frac{h_{j}}{l_{j}-h_{j}}} \geq 0 \\
i=1,2, \mathrm{~L}, n, j=1,2, \mathrm{~L}, m
\end{gathered}
$$

then the bidder $i$ will submit a new bidding with the score $s_{i}^{(t+1)}=S_{\max }^{\text {current }}+D$ and the optimal values $p_{i}^{(t+1)^{*}}$ and $a_{i j}^{(t+1)^{*}}$ are as follows.

$$
\begin{gathered}
a_{i j}^{(t+1)^{*}}=\left(\frac{s_{i} k_{j} h_{j}}{l_{j} w_{j}}\right)^{\frac{1}{l_{j}-h_{j}}}, \\
p_{i}^{(t+1)^{*}}=\sum_{j=1}^{m} w_{j}\left(\frac{s_{i} k_{j} h_{j}}{l_{j} w_{j}}\right)^{\frac{l_{j}}{l_{j}-h_{j}}}-S_{\max }^{\text {current }}-D,
\end{gathered}
$$

where $l_{j} \neq h_{j}, i=1,2, \mathrm{~L}, n, j=1,2, \mathrm{~L}, m$.

(ii) If the following condition is satisfied

$$
\sum_{j=1}^{m} w_{j}\left(\frac{s_{i} k_{j} h_{j}}{l_{j} w_{j}}\right)^{\frac{l_{j}}{l_{j}-h_{j}}}-S_{\max }^{\text {current }}-D-\sum_{j=1}^{m} s_{i} k_{j}\left(\frac{s_{i} k_{j} h_{j}}{l_{j} w_{j}}\right)^{\frac{h_{j}}{l_{j}-h_{j}}}<0
$$

where $l_{j} \neq h_{j}, i=1,2, \mathrm{~L}, n, j=1,2, \mathrm{~L}, m$, then the bidder $i$ will not submit a new bidding, which means the bidder $i$ 's bidding is over. 
Proof. The bidder $i$ 's optimal bidding strategy of the $(t+1)$ th round is equivalent to solve the optimization problem $M_{2}$. Next we give the detail process.

From (8), we have

$$
p_{i}^{(t+1)}=\sum_{j=1}^{m} w_{j}\left(a_{i j}^{(t+1)}\right)^{l_{j}}-S_{\max }^{\text {current }}-D
$$

Substituting (13) into (7), the optimization objective becomes

$\operatorname{Max} u_{s i}^{1}=\sum_{j=1}^{m} w_{j}\left(a_{i j}^{(t+1)}\right)^{l_{j}}-S_{\max }^{\text {current }}-D-\sum_{j=1}^{m} s_{i} k_{j}\left(a_{i j}^{(t+1)}\right)^{h_{j}}$

The goal of Max $u$ is equivalent to $\frac{\partial u_{s i}^{1}}{\partial a_{i j}^{(t+1)}}=0$, i.e.,

$$
\frac{\partial\left[\sum_{j=1}^{m} w_{j}\left(a_{i j}^{(t+1)^{*}}\right)^{l_{j}}-S_{\max }^{\text {current }}-D-\sum_{j=1}^{m} s_{i} k_{j}\left(a_{i j}^{(t+1)^{*}}\right)^{h_{j}}\right]}{\partial a_{i j}^{(t+1)^{*}}}=0
$$

By solving this equation, $a_{i j}^{(t+1)^{*}}$ is obtained as follows.

$$
a_{i j}^{(t+1)^{*}}=\left(\frac{s_{i} k_{j} h_{j}}{l_{j} w_{j}}\right)^{\frac{1}{l_{j}-h_{j}}},
$$

where $l_{j} \neq h_{j}, i=1,2, \mathrm{~L}, n, j=1,2, \mathrm{~L}, m$. Substituting $a_{i j}^{(t+1)^{*}}$ into (8), we obtain

$$
p_{i}^{(t+1)^{*}}=\sum_{j=1}^{m} w_{j}\left(\frac{s_{i} k_{j} h_{j}}{l_{j} w_{j}}\right)^{\frac{l_{j}}{l_{j}-h_{j}}}-S_{\max }^{\text {current }}-D .
$$

In addition, from BRS we know that if a bidder chooses his next new bidding, he must realize the goal of maximizing his current utility. So his $(t+1) t h$ round utility $\quad u_{s i}^{1}=p_{i}^{(t+1)}-\sum_{j=1}^{m} s_{i} k_{j}\left(a_{i j}^{(t+1)}\right)^{h_{j}} \quad$ is nonnegative, together with (11), we can get condition (9) is satisfied. That is, when condition (9) is satisfied, the bidder $i$ will submit new bidding $\left(p_{i}^{(t+1)^{*}}, Q_{i}^{*}, a_{i 1}^{(t+1)^{*}} a_{i 2}^{(t+1)^{*}}, \mathrm{~L}, a_{i m}^{(t+1)^{*}}\right)$ to increase the score and to improve the chances to win the bid.

Otherwise, when condition (12) is satisfied, the rational bidder will not to submit a new bidding. In fact, under condition (12), the bidder $i$ 's utility is negative. This completes the proof of the Proposition 1.

By Proposition 1, we can conclude that the bidder $i$ will achieve the goal of utility maximization, if he submits his multiple rounds of bidding according to (4) and (5).

\section{Feasibility Analysis for Multi-attribute Auction}

In this section, we discuss whether our multi-attribute auction mechanism is a feasible mechanism.

Proposition 2. The multi-attribute auction mechanism given by this paper satisfies incentive compatibility conditions and individual rationality conditions, i.e., it is a feasible mechanism.

Proof. From the scoring function $S_{i}=\sum_{j=1}^{m} w_{j} a_{i j}^{l_{j}}-p_{i}$, we can conclude that the bidders' scores are determined by two factors. The first factor is the quality attribute. The score increases with the increase of the values of quality attributes. The second factor is the price attribute. The score increases with the decrease of the price.

By Proposition 1, the bidder $i$ 's optimal choice of the quality attribute values and price attribute value in each round are given by equation (10) and (11) respectively. From equation (10), the bidder $i$ 's quality attribute values in each round are invariant. So in every round of bidding, bidder $i$ only needs to consider the problem of how to choose the price. From (11),

$$
p_{i}^{(t+1)^{*}}=\sum_{j=1}^{m} w_{j}\left(\frac{s_{i} k_{j} h_{j}}{l_{j} w_{j}}\right)^{\frac{l_{j}}{l_{j}-h_{j}}}-S_{\max }^{\text {current }}-D,
$$

we know that the bidder $i$ 's unit prices in all rounds of bidding are changed from higher to lower. When the condition $p_{i}^{(t+1)}-\sum_{j=1}^{m} s_{i} k_{j}\left(a_{i j}^{(t+1)}\right)^{h_{j}} \geq 0$, i.e., condition (11) is satisfied, the unit price is greater than or equal to the unit cost, the bidder $i$ will continue to enter the next round of bidding to increase opportunities for winning the bid. In this process, the bidders' offer prices will move toward the direction of their true costs. When the unit price is less than the unit cost, the score becomes greater, and the bidder gets more opportunities to win the bid, but the utility is negative. At this moment, the rational bidders will finish their biddings. Therefore, announcing their true costs are the bidders' dominant strategies, which means our multi-attribute auction mechanism satisfies the incentive compatibility conditions.

By the conclusion (ii) in Proposition 1, each bidder's condition for finishing the bidding is as follows. 
$\sum_{j=1}^{m} w_{j}\left(\frac{s_{i} k_{j} h_{j}}{l_{j} w_{j}}\right)^{\frac{l_{j}}{l_{j}-h_{j}}}-S_{\max }^{\text {current }}-D-\sum_{j=1}^{m} s_{i} k_{j}\left(\frac{s_{i} k_{j} h_{j}}{l_{j} w_{j}}\right)^{\frac{h_{j}}{l_{j}-h_{j}}}<0$ which means that each rational bidder will not submit the new bid when the unit price is less than the unit cost, to ensure his utility is greater than zero. That is to say, our multi-attribute auction mechanism satisfies the individual rationality conditions. This completes the proof of the Proposition 2.

\section{Basic Steps of Implementing the Mechanism}

In this section, we give the basic steps to show how to implement the multi-attribute auction mechanism given by this paper to procure divisible goods.

Step 1: The auctioneer (buyer) publishes the basic requirement about the procurement good, and announces the bidding rules (e.g. scoring function $S_{i}=\sum_{j=1}^{m} w_{j} a_{i j}^{l_{j}}-p_{i}$, the reserve price $\bar{p}$, the reserve quality $\underline{a}=\left(\underline{a}_{1}, \underline{a}_{2}, \mathrm{~L}, \underline{a}_{m}\right)$, the reserve score $\underline{S}=\sum_{j=1}^{m} w_{j}\left(\underline{a}_{j}^{l_{j}}\right)-\bar{p}$, the current highest score $S_{\max }^{\text {current }}$, the minimum bid increment $D$, the longest delivery time $T$, the maximum bid quantity $q_{\max }$, and so on) to all bidders (suppliers).

Step 2: All bidders submit multiple rounds biddings one by one in a certain order (such as the order ranked by registration serial numbers). For bidder $i$, when condition (9) is satisfied, he will submit a new bidding with the score $s_{i}^{(t+1)}=S_{\max }^{\text {current }}+D$ and the optimal values $a_{i j}^{(t+1)^{*}}$ and $p_{i}^{(t+1)^{*}}$ given by (10) and (11).

When condition (12) is satisfied, he will not submit a new bidding, which means the bidder $i$ 's bidding is over. When all bidders are no longer to submit new biddings, the whole bidding process is over.

Step 3: When all bidders finish their biddings through multiple rounds bidding, substituting the data information $\left(p_{i}, q_{i}, a_{i j}, Q_{0}, s_{i} w_{j}, l_{j}, h_{j}, q_{\max }\right)$ into model $M_{1}$. By using the software Lingo to solve $M_{1}$, we can determine the winners and the corresponding allowable supply quantity $q_{i}^{*}, i=1,2, \mathrm{~L}, n$. When $q_{i}^{*}>0$, which means bidder $i$ is a winner.

Step 4: The auctioneer and the winners determine the procurement contract. In the contract, for winner $i$, he must supply the $q_{i}^{*}$ units of a divisible good to the auctioneer. The transaction unit price is just the bid price $p_{i}^{*}$ in his final bidding, and the quality level of the supply goods is just the promised quality $\left(a_{i 2}^{*}, \mathrm{~L}, a_{i m}^{*}\right)$ in his final bidding. The auctioneer must pay $p_{i}^{*} q_{i}^{*}$ to the winner $i$.

\section{An application Example}

In this section, we give an application example of electric coal procurement to show how to apply our optimization decision method based on multi-attribute auction and to demonstrate the availability and rationality of this method.

As the country's basic industry, power production has the characteristic of continuous safe stable production, which determines the supply of electric coal is a continuous, balanced and stable, and the electric coal quality (Tvdaf, calorific value, moisture, ash value, ash melting point and coal sulfur value) must meet the requirement of boiler standards and the environmental standards. Electric coal procurement involves many aspects, not only involved in electric coal production, but also involved the transportation of railway, highway and waterway. In order to meet the requirement of the electricity production and to obtain economic benefit, buyers must consider optimal coal procurement strategy under multi-attribute conditions such as quality (Tvdaf, calorific value, moisture, ash value, ash melting point and coal sulfur value), quantity, transport capacity, suppliers' credibility, price, and so on. In practice, the electric coal demand is great in power generation, so a single supplier is often difficult to meet the needs of buyers within a specified time. Therefore, buyers can select multiple suppliers for electric coal supply. In addition, electric coal belongs to continuous homogeneous divisible goods. Therefore, electric coal procurement can be regarded as a kind of multi-attribute procurement of divisible goods.

Now we give a detail application example. It is supposed that a buyer of one power plant uses multiattribute auction mechanism to procure 1000 tons electric coal, he faces five potential suppliers, numbered 1,2,L ,5 . All suppliers are risk neutral, and all want to maximize their expected utilities. Without loss of generality, we select the following four attributes in the auction, i.e., $p$ (price of per ton electric coal, in 
yuan/ton), $q$ (supply quantity, in ton), $A_{1}$ (tvdaf, in percentage), $A_{2}$ (calorific value, in $\mathrm{kcal} / \mathrm{g}$ ), where Tvdaf is the core index to distinguish the combustion characteristic for electric coal. The higher the value of Tvdaf is, the easier it is on fire. Calorific value is an important basis for boiler design. The attribute set is denoted as $A=\left\{p, q, A_{1}, A_{2}\right\}$.

At the beginning of the bidding, the auctioneer announces the bidding rules, concretely, the vdaf in the supplier's bidding must be greater than or equal to $20 \%$, the calorific value in the supplier's bidding must be greater than or equal to $6 \mathrm{kcal} / \mathrm{g}$, the unit bid price is not greater than 285 yuan/ton, the minimum bid increment is $D=1$. Each supplier's bidding must satisfy these requirements. Otherwise, the supplier will be eliminated in the auction.

Let auctioneer's utility of one unit electric coal from the supplier $i$ be

$$
\begin{gathered}
u_{b i}^{1}\left(p_{i}, q_{i}, a_{i 1}, a_{i 2}, \mathrm{~L}, a_{i m}\right)=\sum_{j=1}^{2} w_{j} a_{i j}^{l_{j}}-p_{i}, \\
i=1,2, \mathrm{~L}, 5, j=1,2
\end{gathered}
$$

In this example, we set

$$
l_{1}=0.6, l_{2}=0.9, w_{1}=36, w_{2}=30,
$$

and then the score function can be expressed as

$$
S_{i}=\sum_{j=1}^{2} w_{j} a_{i j}^{l_{j}}-p_{i}=36 a_{i 1}^{0.6}+30 a_{i 2}^{0.9}-p_{i}, i=1,2, \mathrm{~L}, 5 \text {. }
$$

Let the utility of supplier $i$ who submit the unit price $p_{i}$ and gets the one unit of electric coal is

$$
u_{s i}^{1}\left(s_{i}, p_{i}, a_{i 1}, a_{i 2}, \mathrm{~L}, a_{i m}\right)=p_{i}-\sum_{j=1}^{m} s_{i} k_{j} a_{i j}^{h_{j}}
$$

In this example, we set

$$
\begin{gathered}
s_{1}=2.5, s_{2}=2.6, s_{3}=2.7, s_{4}=2.8, s_{5}=2.9 ; \\
k_{1}=1.5, k_{2}=1.6 ; h_{1}=1.1, h_{2}=1.6 .
\end{gathered}
$$

From the above given information, the reserve price is $\bar{p}=190$, and the reserve quantity vector

$$
\underline{a}=\left(\underline{a}_{1}, \underline{a}_{2}\right)=(20,6),
$$

then the reserve score can be obtained as

$$
\underline{S}=\sum_{j=1}^{2} w_{j}\left(\underline{a}_{j}^{l_{j}}\right)-\bar{p}=w_{1} 20^{0.6}+w_{1} 6^{0.9}-290=82.7
$$

Suppose that each supplier submits the following supply quantity according to his production ability within the contractual time and maximum bid quantity $q_{\max }$ given by the buyer (in tons):
$Q_{1}^{*}=420, Q_{2}^{*}=480, Q_{3}^{*}=400, Q_{4}^{*}=460, Q_{5}^{*}=380$.

By Proposition 1, the supplier $i$ 's optimal strategy of quality bidding can be computed by (10), i.e.,

$$
a_{i j}^{*}=\left(\frac{s_{i} k_{j} h_{j}}{l_{j} w_{j}}\right)^{\frac{1}{l_{j}-h_{j}}}, \quad i=1,2, \mathrm{~L}, 5, \quad j=1,2
$$

Substituting $s_{i}, l_{j}, h_{j}, k_{j}, w_{j}$ into $a_{i j}^{*}$, we have

$$
\begin{aligned}
& a_{1}^{*}=\left(a_{11}^{*}, a_{12}^{*}\right)=(27.42,7.82) \\
& a_{2}^{*}=\left(a_{21}^{*}, a_{22}^{*}\right)=(25.35,7.39) \\
& a_{3}^{*}=\left(a_{31}^{*}, a_{32}\right)=(23.51,7.00) \\
& a_{4}^{*}=\left(a_{41}^{*}, a_{42}^{*}\right)=(21.86,6.65) \\
& a_{5}^{*}=\left(a_{51}^{*}, a_{52}^{*}\right)=(20.38,6.32)
\end{aligned}
$$

Clearly, we can see that these values are all greater than the reserve quantity values $\underline{a}=\left(\underline{a}_{1}, \underline{a}_{2}\right)=(20,6)$.

That is, these biddings are all valid.

Based on the above optimal strategies of quantity bidding $a_{i}^{*}, i=1,2, \mathrm{~L}, 5$, each supplier's cost of one unit good can be obtained by

$$
c_{i}^{1}=\sum_{j=1}^{2} c_{i j}\left(s_{i}, a_{i j}^{*}\right)=s_{i} k_{1}\left(a_{i 1}^{*}\right)^{h_{1}}+s_{i} k_{2}\left(a_{i 2}^{*}\right)^{h_{2}}
$$

so we have

$$
\begin{gathered}
c_{1}^{1}=250.60, c_{2}^{1}=238.73, c_{3}^{1}=227.85, \\
c_{4}^{1}=217.83, c_{5}^{1}=208.58
\end{gathered}
$$

According to the conclusions of Proposition 1, the following condition can be used to determine what time to finish bidding for supplier $i$.

$$
u_{s i}^{1}=p_{i}^{*}-\sum_{j=1}^{2} c_{i j}\left(s_{i}, a_{i j}^{*}\right)=p_{i}^{*}-c_{i}^{1} \leq 0, i=1,2, \mathrm{~L}, 5
$$

where $p_{i}^{*}=\sum_{j=1}^{m} w_{j}\left(\frac{s_{i} k_{j} h_{j}}{l_{j} w_{j}}\right)^{\frac{l_{j}}{l_{j}-h_{j}}}-S_{\max }^{\text {current }}-D$.

Now the overall process of suppliers' multiple rounds of bidding is simulated based on basic steps given by section 6 , and the simulation results are listed in Table 1.

By analyzing the data in Table 1, we can get that the supplier 1, supplier 2, supplier 3, supplier 4 and supplier 5 will finish the bidding in the end of $12^{\text {th }}$ round, the $11^{\text {th }}$ round, the $6^{\text {th }}$ round, the $4^{\text {th }}$ round, the $1^{\text {st }}$ round, respectively, and their final optimal bidding strategies are listed as follows.

$$
\left(p_{1}^{*}, Q_{1}^{*}, a_{11}^{*}, a_{12}^{*}\right)=(260.46,420,27.42,7.82), \quad S_{1}=193
$$


$\left(p_{2}^{*}, Q_{2}^{*}, a_{21}^{*}, a_{22}^{*}\right)=(240.00,480,25.35,7.39), \quad S_{2}=192$

$\left(p_{3}^{*}, Q_{3}^{*}, a_{31}^{*}, a_{32}^{*}\right)=(230.31,400,23.51,7.00), \quad S_{3}=182$

$\left(p_{4}^{*}, Q_{4}^{*}, a_{41}^{*}, a_{42}^{*}\right)=(218.19,460,21.86,6.65), \quad S_{4}=176$

$\left(p_{5}^{*}, Q_{5}^{*}, a_{51}^{*}, a_{52}^{*}\right)=(213.46,380,20.38,6.32), \quad S_{5}=164$

Substituting above relative data into model $M_{1}$, we get an optimization problem as follows.

$$
\begin{aligned}
& \max u_{b}=\sum_{i=1}^{5} q_{i}^{*}\left[\sum_{j=1}^{2} w_{j} a_{i j}^{l_{j}}-p_{i}^{*}\right], \\
& \text { s.t. } \\
& \left\{\begin{array}{l}
\sum_{i=1}^{5} q_{i}^{*}=1000 \\
0 \leq q_{i}^{*} \leq Q_{i}^{*}, \quad i=1,2, \mathrm{~L}, 5
\end{array}\right.
\end{aligned}
$$

By using software Lingo 9.0 to solve this optimization problem, we obtain

$$
q_{1}^{*}=420, q_{2}^{*}=480, q_{3}^{*}=100,0, q_{4}^{*}=0, q_{5}^{*}=0 .
$$

From these results, the final allocation scheme is as follows. The supplier 1 will supply 420 tons electric coal to the buyer, the transaction price is $260.46 y u a n /$ ton. The supplier 2 will supply 480 tons electric coal to the buyer, the transaction price is 240 yuan/ton. The supplier 3 will supply 100 tons electric

\begin{tabular}{|c|c|c|c|c|}
\hline Round & Supplier & Bidding $\left(p_{i}^{*}, Q_{i}^{*}, a_{i 1}^{*}, a_{i 2}^{*}\right)$ & Score $S_{i}$ & Whether to submit the bid (Yes or No) \\
\hline \multirow{5}{*}{$\begin{array}{c}\text { The first } \\
\text { round }\end{array}$} & Supplier 1 & $(293.46,420,27.42,7.82)$ & 160 & $u_{s 1}^{1}>0$, Yes \\
\hline & Supplier 2 & $(271.00,480,25.35,7.39)$ & 161 & $u_{s 2}^{1}>0$, Yes \\
\hline & Supplier 3 & $(250.31,400,23.51,7.00)$ & 162 & $u_{s 3}^{1}>0$, Yes \\
\hline & Supplier 4 & $(231.19,460,21.86,6.65)$ & 163 & $u_{s 4}^{1}>0$, Yes \\
\hline & Supplier 5 & $(213.46,380,20.38,6.32)$ & 164 & $u_{s 5}^{1}>0$, Yes \\
\hline \multirow{5}{*}{$\begin{array}{c}\text { The second } \\
\text { round }\end{array}$} & Supplier 1 & $(288.46,420,27.42,7.82)$ & 165 & $u_{s 1}^{1}>0$, Yes \\
\hline & Supplier 2 & $(266.00,480,25.35,7.39)$ & 166 & $u_{s 2}^{1}>0$, Yes \\
\hline & Supplier 3 & $(245.31,400,23.51,7.00)$ & 167 & $u_{s 3}^{1}>0$, Yes \\
\hline & Supplier 4 & $(226.19,460,21.86,6.65)$ & 168 & $u_{s 4}^{1}>0$, Yes \\
\hline & Supplier 5 & $(208.46,380,20.38,6.32)$ & - & $u_{s 5}^{1}<0$, No, bidding is over \\
\hline \multirow{4}{*}{$\begin{array}{c}\text { The third } \\
\text { round }\end{array}$} & Supplier 1 & $(284.46,420,27.42,7.82)$ & 169 & $u_{s 1}^{1}>0$, Yes \\
\hline & Supplier 2 & $(262.00,480,25.35,7.39)$ & 170 & $u_{s 2}^{1}>0$, Yes \\
\hline & Supplier 3 & $(241.31,400,23.51,7.00)$ & 171 & $u_{s 3}^{1}>0$, Yes \\
\hline & Supplier 4 & $(222.19,460,21.86,6.65)$ & 172 & $u_{s 4}^{1}>0$, Yes \\
\hline \multirow{4}{*}{$\begin{array}{l}\text { The } 4^{\text {th }} \\
\text { round }\end{array}$} & Supplier 1 & $(280.46,420,27.42,7.82)$ & 173 & $u_{s 1}^{1}>0$, Yes \\
\hline & Supplier 2 & $(258.00,480,25.35,7.39)$ & 174 & $u_{s 2}^{1}>0$, Yes \\
\hline & Supplier 3 & $(237.31,400,23.51,7.00)$ & 175 & $u_{s 3}^{1}>0$, Yes \\
\hline & Supplier 4 & $(218.19,460,21.86,6.65)$ & 176 & $u_{s 4}^{1}>0$, Yes \\
\hline \multirow{4}{*}{$\begin{array}{l}\text { The } 5^{\text {th }} \\
\text { round }\end{array}$} & Supplier 1 & $(276.46,420,27.42,7.82)$ & 177 & $u_{s 1}^{1}>0$, Yes \\
\hline & Supplier 2 & $(254.00,480,25.35,7.39)$ & 178 & $u_{s 2}^{1}>0$, Yes \\
\hline & Supplier 3 & $(233.31,400,23.51,7.00)$ & 179 & $u_{s 3}^{1}>0$, Yes \\
\hline & Supplier 4 & $(214.19,460,21.86,6.65)$ & - & $u_{s 4}^{1}<0$, No, bidding is over \\
\hline \multirow{3}{*}{$\begin{array}{l}\text { The } 6^{\text {th }} \\
\text { round }\end{array}$} & Supplier 1 & $(273.46,420,27.42,7.82)$ & 180 & $u_{s 1}^{1}>0$, Yes \\
\hline & Supplier 2 & $(251.00,480,25.35,7.39)$ & 181 & $u_{s 2}^{1}>0$, Yes \\
\hline & Supplier 3 & $(230.31,400,23.51,7.00)$ & 182 & $u_{s 3}^{1}>0$, Yes \\
\hline
\end{tabular}
coal to the buyer, the transaction price is 230.31 yuan/ton. The supplier 4 and supplier 5 fail to win the bid.

Table 1. The simulation results of the overall process. 


\begin{tabular}{cllll}
\hline \multirow{2}{*}{ The $^{\text {th }}$} & Supplier 1 & $(270.46,420,27.42,7.82)$ & 183 & $u_{s 1}^{1}>0$, Yes \\
round & Supplier 2 & $(248.00,480,25.35,7.39)$ & 184 & $u_{s 2}^{1}>0$, Yes \\
& Supplier 3 & $(227.31,400,23.51,7.00)$ & - & $u_{s 3}^{1}<0$, No, bidding is over \\
\hline The $8^{\text {th }}$ & Supplier 1 & $(268.46,420,27.42,7.82)$ & 185 & $u_{s 2}^{1}>0$, Yes \\
round & Supplier 2 & $(246.00,480,25.35,7.39)$ & 186 & $u_{s 2}^{1}>0$, Yes \\
\hline The 9 & Supplier 1 & $(266.46,420,27.42,7.82)$ & 187 & $u_{s 1}^{1}>0$, Yes \\
round & Supplier 2 & $(244.00,480,25.35,7.39)$ & 188 & $u_{s 2}^{1}>0$, Yes \\
\hline The 10 & Supplier 1 & $(264.46,420,27.42,7.82)$ & 189 & $u_{s 1}^{1}>0$, Yes \\
\cline { 2 - 5 } round & Supplier 2 & $(242.00,480,25.35,7.39)$ & 190 & $u_{s 2}^{1}>0$, Yes \\
\hline The 1 ${ }^{\text {th }}$ & Supplier 1 & $(262.46,420,27.42,7.82)$ & 191 & $u_{s 1}^{1}>0$, Yes \\
\cline { 2 - 5 } round & Supplier 2 & $(240.00,480,25.35,7.39)$ & 192 & $u_{s 2}^{1}>0$, Yes \\
\hline The 12 & Supplier 1 & $(260.46,420,27.42,7.82)$ & 193 & $u_{s 1}^{1}>0$, Yes \\
round & Supplier 2 & $(238.00,480,25.35,7.39)$ & - & $u_{s 2}^{1}<0$, No, bidding is over
\end{tabular}

\section{Conclusions}

In this paper, an optimization decision method based on multi-attribute auction is proposed for solving the problem of procuring a kind of divisible goods. Compared with the existing procurement decision methods, the contribution of the paper is threefold as follows.

Firstly, traditional procurement methods can not create an incentive and competitive trading environment to induce suppliers to announce their actual information truthfully. In this paper, the traditional resources procurement problem is converted into a multi-attribute procurement auction problem. So it provides a complete freedom competitive environment for trading a kind of divisible goods. This incentive method is can induce the suppliers to announce their actual costs truthfully, and improve the efficiency of the social resources allocation.

Secondly, in the traditional multi-attribute auctions, it is supposed all bidders are symmetrical, and the buyers' utility functions are defined as special functions, and lose the generality features, and the fact that some attributes' marginal production costs of the seller are increasing is not satisfied. In this paper, we suppose all bidders are asymmetrical, and the utility functions of auctioneers and sellers are improved and become more generalized. These results enrich and expand the mechanism design theory of multi-attribute auctions.

Thirdly, in the multi-attribute procurement auction mechanism, a multi-source procurement strategy is presented to select the winner. Under this procurement strategy, the winner can be one, or more. So the procurement process becomes more flexible and more competitive. The buyer has more chances to procure higher quality goods, and also to seek more partners to establish extensive cooperation.

As for other directions for our future research, we think the mechanism design for multi-dimensional auctions, two stages auctions in which the second stage includes negotiations, and how to apply these multiattribute auctions to the fields of real business and industry world, etc, are valuable and interesting issues.

\section{Acknowledgements}

This work is supported by the National Natural Science Foundation of China Grant(No. 71201064), the Natural Science Foundation of Hubei Province to C.J. Rao, the 2012 Excellent Youth Project of Hubei Provincial Department of Education(No. Q20122702), and the Ph.D. Fund of Huanggang Normal University(No. 2011CD257).

\section{References}

1. J. E. Teich, H. Wallenius, J. Wallenius, et al, Emerging multiple issue e-auctions, European Journal of Operational Research 159(1) (2004) 1-16.

2. Y. K. Che, Design competition through multidimensional auctions, Rand Journal Economics 24(4) (1993) 668-680.

3. D. W. Wang, Modeling and Optimization in E-commerce (Science Press, Beijing, 2008).

4. M. Bichler and M. Kaukal, Design and implementation of a brokerage service for electronic procurement, in Proceedings of the 10th International Workshop on Database and Expert Systems Applications, 1999, pp. 618612. 
5. C. J. Rao and Y. Zhao, Mechanism design for optimal auctions of divisible goods, International Journal of Information Technology \& Decision Making 9(5) (2010) 831-845.

6. J. Chen and X. L. Chen, Auction Theory and Internet Auction (Tsinghua University Press, Beijing, 2005).

7. S. L. Liu and M. X. Wang, Multi-attribute procurement auction theory and application: a review with comments, Chinese Journal of Management Science 17(1) (2009) 183192.

8. C. J. Rao and Y. Zhao, Optimal multi-attribute auctions for divisible goods. International Journal of Information Technology \& Decision Making 10(5) (2011) 891-911.

9. C. J. Rao, Y. Zhao and Z. C. Zhang, Multi-attribute auction method based on grey relational degree of hybrid sequences, The Journal of Grey System 21(2) (2009) 175184.

10. M. Bichler, An experimental analysis of multi-attribute auctions, Decision Support Systems 29(3) (2000) 249- 268.

11. S. Thiel, Some evidence on the winner's curse, American Economic Review, 78(5) (1988) 884-895.

12. F. Branco, The design of multi-dimensional auctions, Rand Journal of Economics 28(1) (1997) 63-81.

13. D. Mishra and D. Veeraman, A multi-attribute reverse auction for outsourcing, in Proceedings of the 13th International Workshop on Database and Expert Systems Applications, 2002, pp. 675-679.
14. D. R. Beil and L. M. Wein, An inverse-optimization-based auction mechanism to support a multi-attribute RFQ process, Management Science 49(11) (2003) 1529-1545.

15. E. David, R. Azoulay-Schwartz and S. Kraus, Bidding in sealed-bid and English multi-attribute auctions, Decision Support Systems 42 (2006) 527-556.

16. C. Chen-Ritzo, T. P. Harrison, et al, Better, faster, cheaper: an experimental analysis of a multi-attribute reverse auction mechanism with restricted information feedback, Management Science 51(12) (2005) 1753-1762.

17. G. Perrone, P. Roma and G. Lo Nigro, Designing multiattribute auctions for engineering services procurement in new product development in the automotive context, International Journal of Production Economics 124 (2010) 20-31.

18. A. K. Ray, M. Jenamani, P. K. J. Mohapatra, An efficient reverse auction mechanism for limited supplier base, Electronic Commerce Research and Applications 10(2) (2011) 170-182.

19. G. Karakaya and M. Köksalan, An interactive approach for multi-attribute auctions, Decision Support Systems (2011) 51(2) 299-306.

20. S. L. Liu, J. Li and D. Liu, Multi-attribute procurement auctions with risk averse suppliers, Economics Letters 115(3) (2012) 408-411. 